\title{
Éric-Emmanuel Schmitt et ses maîtres de bonheur : à la croisée des voies littéraire et musicale
}

Romancier, dramaturge et essayiste, Éric-Emmanuel Schmitt est l'auteur d'une œuvre plurielle, empreinte de l'esthétique contemporaine par ses implications intertextuelles, son esthétique syncrétique, le dialogue de la littérature et des arts, ses composantes autobiographiques. Dans plusieurs écrits à éléments autobiographiques, comme le cycle intitulé "Le bruit qui pense ", l'essai Plus tard je serai un enfant et le récit Madame Pylinska et le secret de Chopin, l'auteur révèle la genèse musicale de sa vocation d'écrivain. L'inspiration musicale s'avère ainsi fondatrice pour sa voie littéraire et pour sa voix singulière d'auteur contemporain.

L'objectif de cet article est d'étudier le rôle (trans)formateur de l'apprentissage musical et plus particulièrement de l'initiation à la musique de Mozart, Beethoven et Chopin. Guides spirituels et maîtres à penser, ces compositeurs ont marqué le parcours existentiel et artistique d'Éric-Emmanuel Schmitt. La rencontre salutaire avec Mozart, vécue et décrite par l'écrivain dans Ma vie avec Mozart, induit la naissance de l'amour spirituel et engendre le processus de conversion scripturale et de transmission créatrice. Un autre trope du lien maitre-disciple est analysé à la lumière de l'enseignement de la professeure de piano madame Pylinska, guidant le jeune amateur de musique vers la voie de la création littéraire et la prise de conscience de son talent et de son champ d'expression artistique.

- Antoaneta Robova - maitre de conférences au Département d'Études Romanes de l'Université de Sofia "Saint Clément d'Ohrid ». Adresse de correspondance : Département d'Études Romanes, Université de Sofia "Saint Clément d’Ohrid », boulevard Tsar Osvoboditel 15, bureau 160A, Sofia, Bulgarie ; e-mail : arobova@uni-sofia.bg

ORCID iD : https://orcid.org/0000-0001-7468-7675 


\section{Maîtres de bonheur et maîtres de sagesse ou la genèse musicale d'une voie littéraire}

Par l'étendue de ses intérêts artistiques extralittéraires et la pluralité de ses champs d'expression, englobant le cinéma ${ }^{1}$ et la bande dessinée, mais surtout la musique, Éric-Emmanuel Schmitt pourrait être rattaché à la lignée des "écrivains-artistes » ouverts aux procédés d'hybridation et de métissage, dont l'œuvre porte l'empreinte de l'« esthétique de l'interaction des arts »(Scarpetta, 1980, p. 20). Le rôle privilégié de la musique et de quelques compositeurs dont se détachent les figures magistrales de Mozart et de Beethoven marque et jalonne la trajectoire existentielle et le parcours artistique de l'auteur en lui ouvrant paradoxalement les voies de la littérature et en lui révélant sa voix singulière d'écrivain aux talents multiples.

L’ambivalence identitaire de l'artiste partagé entre les deux pôles de ses pulsions créatrices se reflète dans son prénom composé. Cette particularité onomastique trouve son expression artistique dans l'élaboration d'un mythe personnel aux consonances élégiaques. L'anecdote racontée dans la préface de « Mes maîtres de bonheur » présente l'histoire des jumeaux Éric, futur écrivain, et Emmanuel au destin de musicien, dont l'un se noya. Les parents, ignorant lequel des deux est décédé, décidèrent de rebaptiser le rescapé Éric-Emmanuel. Cette histoire qui " n’est pas véridique, mais elle est tellement vraie » (Schmitt, 2017b, p. 7) réapparaît dans différents écrits ${ }^{3}$ et entretiens pour expliquer les tensions et conflits intérieurs d'un auteur à identité double, partagé entre sa part d'altérité et le mystère de ses prédispositions innées : " Aujourd'hui encore, je m'interroge : si la littérature représente mon art et la musique ma passion, me suis-je trompé ? Ai-je endossé mon destin ? Ou celui de mon frère? » (2017b, p. 7). Le sentiment de deuil symbolique et de perte s'allie à une nostalgie $^{4}$ de l'art de la composition délaissé : « Mes tentatives médiocres et infructueuses de compositeur m’ont confirmé que j’étais Éric plutôt qu'Emmanuel, l'écrivain plutôt

1. Dans le domaine de l'audiovisuel, l'auteur a une expérience de scénariste et de réalisateur.

2. Le lien entre la littérature et les arts visuels ainsi que la « double compétence » des « écrivains-artistes » (Viart, Vercier, 2008, p. 298) sont mis en lumière dans le cas des peintres, photographes et cinéastes. Or, en ce qui concerne Schmitt, la pratique cinématographique est secondaire par rapport à sa vocation littéraire, alors que la musique représente un thème récurrent dans son œuvre. Même si la pratique musicale demeure une passion et non pas un mode d'expression professionnelle, l'auteur n'en est pas moins un «écrivain-musicien » selon la formule de Catherine Lalanne (Schmitt, 2017a, p. 64).

3. Il serait intéressant de noter les différentes versions de cette même histoire constituée en mythe personnel. Sur le site officiel de l'auteur est publiée une variante selon laquelle le frère jumeau n'est pas mort par noyade, mais étouffé sous les couvertures (voir " Autobiographie virtuelle ", https://www. eric-emmanuel-schmitt.com/musique.html). Les variations de cette affabulation fondatrice pour la trajectoire de l'écrivain-musicien ne font que confirmer son statut de mythe personnel et la quête des sens possibles d'une vie en (ré)invention constante.

4. "L'écrivain que je suis est habité par la nostalgie du compositeur que je n’ai pas été » (Schmitt, 2017a, p. 61). 
que le musicien (2017b, p. 8). Un constat qui marque le début de son aventure littéraire se ressourçant aux accords et harmonies du « bruit qui pense ». Schmitt se réfère à la phrase de Victor Hugo «la musique, c'est du bruit qui pense » et met en valeur sa charge méditative : "elle est aussi "du bruit qui fait penser" " (2017b, p. 111). Ce n'est d'ailleurs guère surprenant qu'Éric-Emmanuel Schmitt ait emprunté cette formule métaphorique à Victor Hugo pour en faire le titre de son cycle ${ }^{5}$ consacré aux "musiciens comme maîtres de vie » (2017b, p. 111).

Mozart et Beethoven cristallisent comme deux figures magistrales ayant une importance majeure sur le plan de l'existence et de la création littéraire, deux voix aux résonances multiples dans l'œuvre de Schmitt. Leur présence salvatrice se concrétise dans plusieurs leçons de vie se reflétant sur le mode d'écriture de l'auteur: " Mozart fut mon professeur de bonheur, Beethoven mon maître de joie» (Schmitt, 2017b, p. 9). Mais ils pourraient également être définis comme des maîtres à penser ou " maîtres de sagesse " (2017b, p. 13) dans la mesure où Schmitt les situe à pied d'égalité avec les philosophes qui l'inspirent et les considère à la fois comme des "philosophes sans mots » (2017b, p. 9) et des " guides spirituels » (2017b, p. 111). Les vertus salutaires de la musique et plus particulièrement les propriétés bienfaisantes de l'« Air de la Comtesse » dans Les Noces de Figaro, ayant ranimé la volonté de vivre chez l'adolescent suicidaire, sont décrites dans le chapitre «L’enfant musicien » (2017b, pp. 5164) et dans le roman épistolaire Ma vie avec Mozart. La guérison grâce au pouvoir sublime de la musique annonce le début d'une singulière histoire d'amour et d'une correspondance entre le maître et son disciple. Le disciple mozartien se veut à son tour soigneur des âmes et passeur d'une sagesse heureuse dont les notes et la philosophie indicible se trouvent converties dans le langage de l'écriture. Le genre épistolaire rend possible le régime fictionnel de léchange avec un interlocuteur absent donnant des signes perceptibles par son fidèle disciple aspirant à perpétuer la mission de son maître de bonheur, qui devient progressivement un maître à penser et à créer.

L'auteur avoue avec une note de défi musico-littéraire : "Mon modèle d'écrivain, c'est Mozart » (Schmitt, Hees, 2016). Il revient sur le thème concernant l'influence de la musique de Mozart sur son écriture dans «L'enfant musicien» (Schmitt, 2017b) et s'exprime en nuançant l'idée de la genèse musicale de sa voie littéraire :

Mon écrivain préféré est un musicien! J’ai pétri mon processus créatif de son exemple. Quand j'élabore une phrase, je la pense avec mon esprit mais j'extrais son humeur de mon cœur et la fais résonner dans mon corps. Je produis mon texte comme un musicien sa mélodie, en me servant de mon instrument charnel et en veillant à la vibration sentimentale. (Schmitt, 2017b, p. 61-62).

5. Le cycle contient déjà deux œuvres regroupées dans le recueil thématique Mes maîtres de bonheur: Ma vie avec Mozart et Quand je pense que Beethoven est mort alors que tant de crétins vivent... Mais l'auteur précise : «Suivront bientôt Bach et Schubert... » (Schmitt, 2017b, p. 111). 
Dans ses entretiens et discours métalittéraires, Schmitt n'hésite pas à appliquer des notions musicologiques aux contenus littéraires pour mettre en lumière certains enjeux de son esthétique ouverte au dialogue avec les formes et procédés d'ordre musical. La musicalité littéraire de Schmitt se caractérise par des particularités comme les thèmes à variations, l'attention portée aux tempos, l'économie des moyens. L'auteur se situe dans la continuité esthétique de Mozart et s'en réclame au point de se définir comme une figure ambivalente d'écrivain disciple mozartien. Par le lien à son maitre, Schmitt se situe dans la logique d'une philiation ou filiation affective et spirituelle faite d'amour ${ }^{6}$, de gratitude et d'admiration. Le but du disciple mozartien est de perpétuer le geste de transmission et de poursuivre la continuité. Cette philiation se révèle réconfortante et salvatrice : "Mon but s'avoue autant existentiel que culturel. Introduire à Mozart, certes, mais surtout guérir par Mozart, réconforter par Mozart, convertir à la joie par Mozart. L'art nous aide à vivre » (Schmitt, 2017a, p. 60).

\section{L'aventure esthétique du disciple mozartien}

Ma vie avec Mozart représente un livre hybride car il entremêle la littérature et la musique en créant un dialogue ${ }^{7}$ constant entre les deux arts au fil des pages. Par ailleurs, c'est un roman épistolaire qui enchevêtre l'écriture de soi, les digressions essayistiques et multiplie les citations musicales et les commentaires. Ce journal intime, revêtant la forme d'une correspondance avec Mozart, maître et soigneur salvateur, décrit sur le mode de la confidence la transformation d'une aventure mortelle en aventure esthétique ${ }^{8}$ et la prise de conscience d'une mission de disciple et de passeur de valeurs esthétiques. L'aventure devenue mortelle de la traversée périlleuse de l'adolescence en mal de repères stables et la quête d'une identité au-delà des pulsions suicidaires se mue en aventure esthétique grâce au pouvoir envoûtant de la musique. En même temps, l'aventure esthétique va de pair avec une histoire d'amour peu conventionnelle, car faite d'amour spirituel ${ }^{9}$ envers un maître immortel, transformant un « destin clos » en " destinée ${ }^{10}$ ouverte et en voie vers l'expression littéraire et artistique.

6. Philia, un des mots grecs pour désigner les différents types d'amour, entre dans la composition de ce mot-valise, mais nous allons voir ultérieurement le rôle joué par l'« éros » selon la définition de Georges Steiner dans le lien maître-disciple qui unit Mozart et Schmitt.

7. Le livre Ma vie avec Mozart a été créé à l'occasion du $250^{\mathrm{e}}$ anniversaire de la naissance du grand compositeur. Le dialogue des lettres et des morceaux de musique en guise de réponse au-delà du temps et des mots a connu une transposition scénique (Palais des Beaux-Arts, Bruxelles, 25/10/05).

8. Vladimir Jankélévitch examine trois formes de l'aventure : mortelle, esthétique et amoureuse (Jankélévitch, 2017, p. 9-58). Ayant échappé à ses pulsions autodestructrices grâce à la beauté de la musique, le jeune Éric-Emmanuel Schmitt se tourne vers l'avènement de son aventure esthétique, faisant de son existence une matière romanesque et lui conférant un « sens esthétique » (Jankélévitch, 2017, p. 31).

9. Max Scheler évoque trois formes d'amour : vital, psychique et spirituel. L'amour spirituel représente la forme supérieure d'amour (Scheler, 1950).

10. Au sujet de la distinction entre « destin » et « destinée » : voir Jankélévitch (2017, p. 41-43). 
L'illumination esthétique et la révélation d'une passion salutaire pour la musique a lieu lors d'une répétition des Noces de Figaro à l'opéra de Lyon qui déclenche le processus de (trans)formation chez le jeune homme reprenant goût à la vie. Cet épisode crucial est décrit dans le bref récit rétrospectif à la première personne qui introduit le lecteur aux lettres constituant l'ensemble de la correspondance très personnelle avec Mozart. Le mal de vivre et l'obsession de la mort hantant le futur écrivain se dissipent et se transforment progressivement en joie de vivre et passion mélomane sous l'effet vivifiant du ravissement musical :

Voilà ce que disait l'adolescent qui, quelques minutes auparavant, voulait s'ouvrir les veines. Mozart m'avait sauvé : on ne quitte pas un univers où l'on peut entendre de si belles choses, on ne se suicide pas sur une terre qui porte ces fruits, et d'autres fruits semblables.

La guérison par la beauté... Aucun psychologue n’aurait songé sans doute à m’appliquer ce traitement.

Mozart l'a inventé et me l’a administré.

Telle une alouette filant vers le ciel, je sortais des ténèbres, je gagnais l'azur.

Je m'y refugie souvent. (Schmitt, 2017b, p. 22)

L’adolescent désespéré et mélancolique s'imprègne ainsi de l'expérience amoureuse de la musique qui l'élève et le transfigure. L'image de l'alouette et l'élan vers l'azur du ciel, au-delà des ténèbres de la mélancolie, reflètent le franchissement de la période d'introspection autodestructrice et l'ouverture aux bienfaits de l'art et à l'enseignement de son guide spirituel. Le jeune homme découvre les vertus purificatrices de la musique classique aux effets cathartiques. Il se voue ainsi à un apprentissage affectif, existentiel et musico-scriptural, lors duquel il est guidé par Mozart, son " maitre de sagesse " qui lui enseigne " des choses si rares, lémerveillement, la douceur, la sérénité, la joie... » (Schmitt, 2017b, p. 13).

La rencontre soudaine et fatidique avec le maître survient par l'intermédiaire de son legs musical et donne lieu à une expérience initiatique et à un "coup de foudre ${ }^{11}$ esthétique et affectif. Ce ravissement musical éveille les sens du jeune homme à la sensibilité exacerbée et guérit son âme tourmentée et spleenétique. L'« amour doublé de reconnaissance» (Schmitt, 2017b, p. 13) de l'écrivain pour son maître commence avec les premières notes de l'" Air de la Comtesse » et le jeune homme de quinze ans qu'il était ressent un véritable " choc amoureux ", ainsi que les effets de "l'amour naissant "(Alberoni, 1981). L'hommage d'Éric-Emmanuel Schmitt à son maître prend d'ailleurs une forme musicale plus tangible par l'intégration de renvois

11. La naissance de la passion musicale et de l'amour pour Mozart est décrite grâce au lexique relatif au discours amoureux. Nous trouvons le mot « coup de foudre » dans l'entretien de Catherine Lalanne avec Schmitt : "J'éprouvai donc un coup de foudre à retardement » (2017a, p. 57) et dans Ma vie avec Mozart : « Un coup de foudre, c'est aussi mystérieux en art qu'en amour » (2017b, p. 24). 
explicites aux œuvres mozartiennes citées dans le livre. Le dialogue entre les mots et les notes, le maître et son disciple se trouve amplifié grâce à ces indications musicales aux caractères manuscrits. Les seize extraits d'œuvres musicales qui jalonnent Ma vie avec Mozart représentent les réponses et les conseils du compositeur immortel qui se montre compréhensif envers son disciple et sensible à ses questionnements et confessions épistolaires.

"C'est lui qui a commencé notre correspondance » (Schmitt, 2017b, p. 13), annonce l'incipit de cette œuvre singulière dédiée à l'art comme mode de (sur)vie et célébrant un dialogue rendu possible par la magie de l'échange entre musique et littérature et par l'intensité soudaine d'un coup de foudre inhabituel. Il marque le début d'une histoire d'amour, de partage et de quête de la voie d'expression artistique d'un «créateur joyeux " (Schmitt, 2017a, p. 79) aux multiples intérêts. La première lettre de la correspondance avec Mozart est inspirée de l' " Air de la Comtesse " des Noces de Figaro. Sa valeur inaugurale est doublée d'une teneur émotive et poétique d'épanchement lyrique, car cette déclaration d'amour passionnée et philosophique comporte aussi un aspect prophétique :

Tu as donc été, Mozart, un coup de foudre à retardement. [...]

Plutôt qu'une découverte, c'est une révélation.

Révélation de quoi ? Ni du passé, ni du présent. Révélation du futur...

Cela relève de la prescience, le coup de foudre... La durée se plisse, se tord, et voilà qu'en une seconde jaillit l'avenir. Nous voyageons dans le temps. Nous accédons non à la mémoire du passé mais à la mémoire de demain. « Voici le grand amour des prochaines années que j’ai à vivre. » (Schmitt, 2017b, p. 24)

Le coup de foudre est perçu comme un rapport au temps et s'apparente ainsi à une aventure fluide et imprévisible, vécue sur le mode de la futurition, associée au « surgissement de l'avenir » (Jankélévitch, 2017, p. 7). L'aventure esthétique est appréhendée comme une aventure amoureuse qui rompt le cours habituel de l'existence et brise sa morne monotonie pour y introduire l'étincelle de l'espoir et la joie de l'ouverture vers l'altérité. Selon les propos de Jankélévitch, l'aventure amoureuse « apparaît $[\ldots]$ comme une parenthèse à l'intérieur du vécu, comme une sorte de madrigal ou de poème en vers interpolé au milieu du texte prosaïque et sérieux de l'existence » (2017, p. 44). Mais le coup de foudre ou l'aventure esthético-amoureuse n'est que l'occasion de l'esquisse d'un avenir fait d'apprentissage et de partage par la conversion du coup de foudre en une "longue et belle histoire ensemble» (Schmitt, 2017b, p. 24).

Les vertus thérapeutiques de la musique de Mozart transforment le jeune homme en disciple mozartien, désirant assimiler et transmettre la leçon de bonheur de son maitre et perpétuer son génie en traduisant sa philosophie ineffable des notes et harmonies dans le langage des mots. L'illumination musicale déclenche l'aventure esthétique de l'écriture alors que l'état naissant de l'amour insuffle aux mots la poésie, 
la sagesse et la mélodie des opus mozartiens. L'écrivain transcende son amertume de l'absence de talent pour la composition musicale et dépasse le sentiment de frustration pour trouver sa vocation et son accomplissent dans le domaine des lettres et devenir passeur de la philosophie mozartienne ${ }^{12}$. Schmitt met en valeur ses prédilections musicales et l'influence majeure des compositeurs comme maîtres à penser et modèles d'écriture :

J'entretiens une conversation constante avec la musique, l'art que je place au-dessus de tous les autres. Avec Mozart d'abord, mais aussi avec Schubert, Bach, Beethoven, Chopin, Bizet, Debussy, Messiaen... Je cherche leurs notes sous ma plume, je leur offre mes phrases à l'occasion d'une chanson ou d'un opéra. Oui, j'invente des livres pour qu'on entende mieux la musique. (Schmitt, 2017a, p. 61)

La présence du maitre demeure pourtant médiée par ses œuvres alors que la réciprocité des échanges décrits dans Ma vie avec Mozart est fondée sur la construction mentale d'une communication par signes secrets et morceaux de musique. Mais cet échange imaginaire consacre le rôle privilégié de Mozart dans le parcours existentiel et artistique du disciple qui se tourne vers son maitre dans des moments cruciaux. L’identification à des personnages comme Chérubin ou Don Juan marque différentes étapes du passage à l'âge adulte et la construction identitaire du jeune homme épris de musique. La lettre du trentenaire reflète les interrogations d'un homme cultivé qui doute des qualités de la musique d'un compositeur " pas assez élitaire " (Schmitt, 2017b, p. 33). Mais il retrouve la beauté de l'art du maître à la faveur de l'ariette touchante de l'«Air de Barberine» qui lui apporte une seconde guérison, celle du conformisme intellectuel perçu comme « une maladie de jeunesse : la sophistication doublée d'une hypertrophie de la pensée » (2017b, p. 36). Après ces retrouvailles, les lettres se suivent pareilles aux décennies qui s'écoulent. Le motet exquis Ave verum corpus à l'occasion de Noël représente un "moment sacré » et éveille un élan spirituel chez le futur écrivain sensible à l'invisible.

Les vertus consolatrices de la musique accompagnent l'auteur aux moments difficiles de perte d'êtres chers et l'éclairent de précieuses leçons de sagesse que le lecteur attentif pourrait déceler inscrites en filigrane dans ses œuvres. Ainsi du Concerto pour clarinette qui lui suggère: «Accepter l'inévitable tristesse. Consentir au tragique de l'existence " (Schmitt 2017b, p. 47) ou d'Une petite musique de nuit qui respire l'optimisme au-delà des douleurs de l'existence. Certaines lettres acquièrent d'ailleurs la force de manifestes professant un art de la continuité au lieu de la rupture et exprimant le désir de l'écrivain de se situer dans la lignée de son maittre : "Je voudrais

12. À la question de Catherine Lalanne portant sur sa "profession de foi d'écrivain » et le fait d'avoir "pris la plume pour mettre en mots sa musique », l'auteur répond : « rien ne m'émeut davantage qu'un lecteur qui m’annonce que je joue dans sa vie le rôle que Mozart a joué dans la mienne » (Schmitt, 2017a, p. 60). 
te rejoindre dans l'idéal d'un art simple, accessible, qui charme d'abord, bouleverse ensuite. [...] Il nous faut plaire avant tout, mais plaire sans complaire, [...] en élevant, pas en abaissant » (2017b, p. 70). La fable de l'ineffable prend corps au fil des lettres, pour livrer les manifestations d'une fusion esthétique et d'une expérience tissant et consacrant ce lien très personnel fait d'admiration et de désir de connaissance et de naissance à la beauté de l'art sublime de la musique. Engagé sur les traces de son guide spirituel, le jeune homme réalise la conversion de son élan musical en élan vital et littéraire.

L'aventure esthético-amoureuse dynamise l'échange perçu comme authentique même s'il n'existe que dans sa quintessence de construction scripturale et de communication abstraite, basée sur des synchronicités et des lectures de signes. La première lettre met en valeur la réciprocité des interactions avec la figure magistrale et le rôle de guide existentiel et spirituel qui lui incombe. Le lien de philiation et de continuité créatrice est mis en valeur par le ton de confidence adopté par le sujet écrivant et par le tutoiement exprimant le sentiment de proximité affective : "Lorsque tu m'as envoyé ta lettre, outre ta musique, j'ai reçu l'assurance que nous allions avoir une longue et belle histoire ensemble, que, mon existence entière, tu m'accompagnerais, tu me suivrais, tu me guiderais, tu me glisserais des confidences, tu m'amuserais, tu me consolerais» (Schmitt, 2017b, pp. 24-25). L'expérience esthétique inaugure la voie vers la vocation d'écrivain et se fonde sur un lien maitre-disciple se rapprochant de la troisième catégorie de la taxinomie ${ }^{13}$ de Steiner : " celle de l'échange, d'un éros fait de confiance réciproque et, en vérité, d'amour " (2003, p. 12). Même si cette « osmose» $(2003$, p. 12) peut paraitre illusoire et si l'échange semble cristalliser dans la diégèse poétique d'une confession autobiographique, la particularité de cet amour est de compenser l'unilatéralité de l'apprentissage par l'ouverture du lien vers la continuité de la philiation et de la transmission de la sagesse du bonheur.

\section{La musique humaniste de Beethoven}

Le dialogue entre les mots et les compositions musicales, ainsi que l'hommage à un maître de bonheur et de vie se poursuit dans l'essai Quand je pense que Beethoven est mort alors que tant de crétins vivent... Il est intéressant de relever certaines parentés entre les deux livres du cycle "Le bruit qui pense " dont l'évolution mouvementée de l'histoire d'amour, de sagesse et de philiation. Même s'il est impossible d'évoquer des épisodes de trahison du maître par le disciple, des périodes de doute marquent l'apprentissage du jeune Éric-Emmanuel Schmitt ayant assimilé certaines

13. Steiner distingue trois "structures de relations" (2003, p. 11) maitre-disciple : le scénario de destruction du disciple par le maitre, le cas de trahison du maître par le disciple et le lien de dialogue et d'échanges enrichissants formant le paradigme de "l'arc électrique de la foi partagée et de la paternité » (2003, p. 136). 
tendances de pensée opposant art populaire et art élitaire et dévalorisant les œuvres de Mozart ou connaissant des baisses d'intérêt pour la musique de Beethoven. Mais au lieu de se solder par un acte de rejet et de rupture avec la figure du maître, ces moments de distanciation critique engendrent une méditation sur des questions fondamentales comme les antinomies art populaire-art noble, rupture-continuité, modes-tradition ou snobisme-humanisme et pessimisme-optimisme. Si George Steiner affirme que "dans toute entreprise humaine ou presque, l'apprenti se fait critique, négateur ou rival de son maître » (2003, p. 137), le disciple mozartien et admirateur de l'œuvre humaniste et de «la sagesse de la joie » (Schmitt, 2017b, 168) de Beethoven surmonte les moments de remise en question de l'autorité de ses "maîtres de bonheur » en situant son écriture et sa créativité dans leur sillage sans trahir leur testament de maîtres à penser et à créer. Or, la posture du disciple mozartien qu'est Éric-Emmanuel Schmitt suppose la fidélité ${ }^{14}$ et la réinvention singulière des leçons des maîtres, sur le mode de la conversion scripturale et de l'inspiration esthétique et philosophique.

L'opus essayistique consacré à Beethoven a une forme originale enchevêtrant les éléments autobiographiques, les digressions dédiées à un discours sur l'esthétique musicale et littéraire et les envolées lyriques de la tentative de traduction de la matière musicale dans le langage d'une poésie en prose. En ce sens, le mot opus que nous utilisons pour désigner cette œuvre brève reflète ses particularités musico-littéraires d'une écriture inspirée, nourrie et empreinte de musique classique et ses fonctions d'hommage aux maitres compositeurs. Le fil conducteur de la narration rétrospective à la première personne du singulier retrace le rôle joué par Beethoven comme maitre de vie et assure le cadre narratif des chapitres essayistiques et philosophiques au cœur du livre. L'incipit amène le sujet de la " cohabitation " (Schmitt, 2017b, p. 120) ou de la «relation » (2017b, p. 122) entre le jeune homme et le grand compositeur sur un ton de confidence et situe la fréquentation de la musique de Beethoven dans la logique supérieure d'une expérience existentielle de partage et d'apprentissage : «Entre Beethoven et moi, ce fut une histoire brève mais forte (2017b, p. 113).

La partie rétrospective relate au passé simple les épreuves au fil de l'histoire du compositeur et de son disciple, alors que les digressions essayistiques se déploient au présent. Les références manuscrites aux compositions musicales accompagnent et engendrent le texte. Il présente d'ailleurs un aspect fragmentaire et éclectique reflétant son caractère autobiographique, car selon les mots de l'auteur : " Le piège d'un récit autobiographique consiste à imposer un ordre à des réalités fragmentaires, dissociées $[\ldots]$ et, dès lors, ne plus respecter les réseaux subtils, complexes, anachroniques, sécables, qui tissent l'étoffe d’un destin» (Schmitt, 2017b, p. 145-146).

14. Il serait intéressant d'évoquer l'opinion que les vrais disciples se situent dans un « rapport ambivalent de fidélité et de sacrilège » (Noacco, Bonnet, Marot et Orfanos, 2013, p. 324), mais dans le cas de Schmitt le sacrilège de la rupture novatrice n'a pas lieu en raison des interférences entre musique et littérature et de l'apprentissage existentiel et indirect de la philosophie de ses « maîtres de bonheur ». 
Le récit de l'histoire des cinq ans d'apprentissage grâce à la musique de Beethoven, la séparation à la fin de l'adolescence du disciple et les retrouvailles vingt ans plus tard permet au narrateur de réfléchir sur les particularités de la musique et de la sagesse de Beethoven. L'auteur dresse un parallèle entre Mozart et Beethoven, ses deux maîtres, pour mettre en valeur leurs mérites et les différentes facettes de leur génie. Ainsi insiste-t-il sur la leçon d'hérö̈sme, d'humanisme et d'optimisme reçue du compositeur, qui a su créer des œuvres sublimes même s'il était frappé de surdité et a écrit l'Hymne à la joie, son chef d'œuvre professant une sagesse de la joie : " Humanisme, courage, culte de la hauteur, choix de la joie : voilà les quatre propositions de Beethoven " (2017b, p. 152). S'inscrivant ainsi dans la continuité philosophique de son maitre, Éric-Emmanuel Schmitt est l'auteur d'une œuvre placée sous le signe de sa vision lucide et optimiste de l'existence. Il (re)génère ainsi dans le langage de la littérature le crédo humaniste du maître qu'il appelle « credo de l'optimisme moderne » et formule vers la fin de cet hommage à Beethoven qui l'a aidé à « concevoir un humanisme moderne, un optimisme qui concilie sens du tragique et espoir en l'avenir » (2017b, p. 167).

Dans cet opus, célébrant le génie de Beethoven à travers le prisme de l'apprentissage artistique d'un jeune homme amoureux de musique et ouvert aux modulations spirituelles de la musicalité, se détache une figure d'importance majeure : la professeure de piano et médiatrice de la voix du grand maître et compositeur Beethoven. Madame Vo Than Loc, dont la pratique pédagogique repose sur des méthodes traditionnelles et une approche basée sur la discipline et l'autorité, se distingue par un tempérament impérieux et intransigeant. Le perfectionnisme autoritaire de la professeure perçue par son élève comme un « dragon malveillant» (Schmit, 2017b, p. 118) s'est pourtant atténué avec le temps. L'ancienne cantatrice a progressivement adopté une méthode d'enseignement plus souple qui favorise la découverte personnelle et les échanges. Ce changement de l'approche pédagogique intervient à la suite d'une prise de conscience concernant le penchant artistique de son élève : «Elle devina que, dans le piano, ce n'était pas le piano que j'aimais, mais la musique » (2017b, p. 118).

La valeur initiatique des leçons de piano acquiert une importance cruciale lorsque le jeune amateur de musique propose à sa professeure les Ouvertures de Beethoven. L'épisode de la découverte de l'Ouverture de Coriolan déclenche le processus d'initiation du futur écrivain-musicien à la beauté de cet art spirituel et fait naître le titre de l'opus qui constitue aussi un leitmotiv dans ce récit d'apprentissage. La phrase percutante du titre représente la citation d'une réplique de madame Vo Than Loc, révoltée contre le destin tragique du génie et catégorique dans ses convictions au sujet de l'injustice du sort qui s'est abattu sur le grand compositeur frappé de surdité et d'une mort précoce. Avec l'aide de l'enseignante, le jeune élève découvre Beethoven et l'importance vitale de l'art conférant sens et essence à l'existence et susceptible d'ouvrir la voie vers l'immortalité. Grâce à cette étape de la formation fondatrice pour le futur écrivain, cristallise la prise de conscience de sa vocation créatrice : «Oui, je créerai à mon tour !» (Schmitt, 2017b, p. 126). Si la rencontre avec Beethoven et le chemine- 
ment vers la découverte d'une vocation est à la base de cet opus essayistique, la scène des retrouvailles symboliques avec le compositeur au musée Ny Carlsberg Glyptotek a fait naître l'idée de la pièce de théâtre Kiki van Beethoven. Éric-Emmanuel Schmitt admire l'héroïsme, le génie et l'énergie créatrice de son maître qui lui a transmis la sagesse de la joie malgré les adversités, l'esprit humaniste et la tonalité optimiste de son œuvre : "J'ai écrit Quand je pense que Beethoven est mort alors que tant de crétins vivent... pour manifester ma gratitude à ce géant malmené par le sort qui a choisi envers et contre tout le camp de la vie» (2017a, p. 88-89).

\section{Chopin et le rôle (trans)formateur des leçons de piano}

Paru en 2018, le récit intitulé Madame Pylinska et le secret de Chopin voit le jour comme le septième volet du Cycle de l'invisible, bien qu'il s'inscrive dans la continuité thématique du cycle «Le bruit qui pense » en modulant le «chant de remerciement aux musiciens » (Schmitt, 2017b, p. 9) évoqué dans la préface de Mes maitres de bonheur. L'auteur motive ce choix en expliquant que son éditeur le lui a suggéré " parce que c'est un conte initiatique » (Schmitt, 2018b, 11:06-11:16) et il a accepté sa suggestion car la musique dans ce livre représente « un adjuvant spirituel, [...] une aide spirituelle » (2018b, 11:20-11:30). Le livre, qui représente un récit d'initiation et d'apprentissage, consacre le rôle des musiciens comme guides et maitres spirituels qui professent des « leçons de philosophie » (2018b, 12:15), sont sacralisés par leur(s) disciple $(s)^{15}$ et se révèlent dignes d'une profonde vénération.

Dans ce récit à la première personne, le grand compositeur qui éveille l'admiration et la fascination du jeune Éric-Emmanuel est Chopin, alors que les figures de passeurs de la philosophie musicale de Chopin sont tante Aimée et la professeure de piano madame Pylinska. Le garçon de huit ans, considérant d'abord le piano comme un " intrus », est initié à la beauté exquise de la musique de Chopin par sa tante pianiste. La scène de la révélation de la musique du compositeur et pianiste de génie est décrite dans un langage poétique recréant en mots l'effet d'une musique synesthésique. Cette expérience esthétique fait naître des perceptions sensorielles différentes allant au-delà de l'auditif pour rejoindre les registres tactile et visuel et revêtir une signification mystérieuse à percer :

Au milieu du salon ensoleillé, un nouveau monde avait surgi, un ailleurs lumineux flottant en nappes, paisible, secret, ondoyant, qui nous figeait et nous rendait attentifs. À quoi ? Je l'ignorais. Un événement extraordinaire venait de se dérouler, l'efflorescence d'un univers parallèle, l'épiphanie d'une manière d'exister différente, dense et éthérée,

15. Madame Pylinska se définit comme « monothéiste » : "Je n'aime qu'un compositeur : Chopin » (Schmitt, 2018a, p. 59), alors que le jeune protagoniste se déclare " polythéiste » et présente la liste des divinités musicales qu'il révère : « Je vénère plusieurs dieux : Bach, Mozart... » (2018a, p. 59). 
riche et volatile, frêle et forte, laquelle, tout en se donnant, conservait la profondeur d'un mystère. (Schmitt, 2018a, p. 11)

Émerveillé par cette beauté et animé par le désir de percer l'énigme de Chopin, l'enfant décide de prendre des cours de piano. Sa première professeure Mme Vo Than $\operatorname{Loc}^{16}$ n'arrive pourtant pas à transmettre à son élève le savoir-faire nécessaire pour la bonne interprétation de la musique de Chopin. Ainsi, devenu étudiant à Paris, le jeune Éric-Emmanuel continue son apprentissage du piano chez « une certaine madame Pylinska, auréolée d'une excellente réputation, Polonaise émigrée à Paris » (Schmitt, 2018a, p. 15). Dans ce récit d'apprentissage, décrivant la révélation d'une vocation littéraire par la voie de l'éveil des sens et de la sensibilisation à l'art du Chopin, madame Pylinska remplit la fonction actantielle de l'adjuvante lors de la quête cognitive et esthétique du héros. Le nom de Chopin, prononcé par le futur élève, fonctionne comme une formule magique ouvrant la voie vers son " initiation suprême " (2018a, p. 13) par une enseignante compétente et exigeante. En effet, la professeure de piano, qui voue un culte au grand maitre de la période romantique, accepte la mission de transmettre ses connaissances et son art pianistique au jeune étudiant en philosophie sensible à la poésie de la musique de Chopin.

La (trans)formation du narrateur, cherchant à percer le secret de Chopin, passe par plusieurs épreuves constituant un rite insolite d'initiation et une éducation sensorielle, affective et intellectuelle. Le lecteur découvre la méthode complexe et saugrenue de la professeure qui combine plusieurs des « supports de transmission entre maître et disciple - la parole, l'écriture, la musique, le geste et le silence " (Névot, 2013, p. 11). Les exercices visant à solliciter les sens, à éveiller la sensibilité créatrice et à aider l'élève à prendre conscience de sa véritable vocation relèvent de la pratique d'une maïeutique pédagogique révélant à l'élève la quintessence de son talent. Des exercices et épreuves comme l'expérience physique des vibrations de la musique, cueillir des fleurs sans laisser tomber la rosée, faire des ronds dans l'eau, écouter le silence, explorer les effets du vent dans les arbres, faire l'amour en regardant sa partenaire dans les yeux composent une méthode de formation particulière. Cette formation à la transformation vise à l'éveil à la créativité par la mobilisation de l'attention, le développement du goût des nuances et de la douceur des sensations, par l'illumination du sentiment amoureux et la révélation de la philosophie de la musique et de la musicalité de la littérature pour encourager l'avènement d'une écriture nourrie des leçons de musique et de vie.

Même si la méthode de madame Pylinska n'est point traditionnelle, sa posture d'enseignante inspire une distance respectueuse alors que l'esprit de ses leçons évoque la métaphore de l'" apprivoisement constant " qui se trouve " associé à une double métamorphose » (Constantinescu, 2009, p. 528). L'élève prend conscience

16. Nous avons déjà évoqué ce personnage de l'essai romancé Quand je pense que Beethoven est mort alors que tant de crétins vivent... 
de son avenir d'écrivain et transpose dans son écriture le sens de la délicatesse et des nuances, la douceur et la dextérité d'esprit, les ondulations de la sensibilité et les sens du silence grâce au bilan dressé par sa professeure : «Vous jouez et vous jouerez très bien Chopin. Mais pas sur un piano " (Schmitt, 2018a, p. 113). Après avoir perçu et suggéré à son élève la " porte singulière » (2018a, p. 113) de son talent ou la voie vers sa véritable vocation esthétique, Mme Pylinska prend la décision de rentrer dans sa patrie - le pays où naquit son compositeur vénéré et grand maître de musique. Le dernier chapitre de ce conte initiatique décrit les retrouvailles en Pologne, une trentaine d'années plus tard, de l'écrivain et de sa professeure de piano qui l'accueille les bras ouverts et conclut que «c'était très émouvant, la bonne volonté chez cet homme, une sorte d'hommage rendu à ce qui le dépassait et lui demeurait inaccessible » (2018a, p. 117).

Écrivain contemporain à l'identité artistique ambivalente partagée entre littérature et musique, Éric-Emmanuel Schmitt est l'auteur d'une œuvre à unité thématique forte, mise en valeur par un souci d’organisation en cycles. Il révèle la genèse musicale de son aventure esthétique et l'avènement de sa vocation d'écrivain en soulignant le rôle des figures magistrales des compositeurs ou bien en décrivant la maïeutique pédagogique mise en œuvre par ses professeures de piano. La musique cristallise comme un des topiques schmittiens majeurs et l'auteur rend hommage à ses « maîtres de bonheur " dans son œuvre pour exprimer sa fidélité à leur testament artistique et philosophique. Le disciple mozartien réinvente sa vocation et transpose dans son œuvre littéraire certaines particularités de la sagesse humaniste de ses maîtres à penser et à créer en s'inscrivant dans une logique de philiation ou filiation affective, spirituelle et esthétique.

\section{RÉFÉRENCES}

Alberoni, F. (1981). Le Choc amoureux. Recherches sur l'état naissant de l'amour (J. RaoulDuval et T. Matteucci-Lombardi trad.). Paris : Éditions Ramsay.

Constantinescu, M. (2009). Postface. Apprivoisement de l'autre, (re)construction de soi. Dans

V. Deshoulières. et M. Constantinescu (dir.), Les Funambules de l'affection. Maîtres et disciples (p. 527-529). Clermont-Ferrand : Presses universitaires Blaise Pascal.

Jankélévitch, V. (2017). L'Aventure, l'ennui et le sérieux. Présentation par L. Barillas, P.-A. Guinfolleau et F. Worms. Paris : Flammarion.

Névot, A. (2013). Introduction. Au fil de la transmission. Un corps à corps maître-disciple. Dans A. Névot (dir.), De l'Un à l'autre. Maîtres et disciples (p. 9-34). Paris : CNRS.

Noacco, C., Bonnet, C., Marot, P. et Orfanos, C. (2013). Conclusion. Dans C. Noacco, C. Bonnet, P. Marot. et C. Orfanos (dir.), Figures du maître. De l'autorité à l'autonomie (p. 323-327). Rennes: PU Rennes.

Scarpetta, G. (1985). L'Impureté. Paris : Grasset.

Scheler, M. (1950). Nature et formes de la sympathie. Contribution à l’étude des lois de la vie émotionnelle (M. Lefebvre trad.). Paris : Payot. 
Schmitt, É.-E., Hees, J.-L. (2016). Entretien avec Éric-Emmanuel Schmitt par Jean-Luc Hees. Paris : Audiolib.

Schmitt, É.-E. (2017a). Plus tard, je serai un enfant. Entretiens avec Catherine Lalanne. Paris : Bayard.

Schmitt, É.-E. (2017b). Mes Maîtres de bonheur. Paris : Librairie générale française.

Schmitt, É.-E. (2018a). Madame Pylinska et le secret de Chopin. Paris : Albin Michel.

Schmitt, É.-E. (2018b). Conférence d'Éric-Emmanuel Schmitt - Madame Pylinska et le secret de Chopin. Entretien avec l'auteur Éric-Emmanuel Schmitt au Centre des Arts d'Enghien les Bains. Récupéré de https://www.youtube.com/watch?v=GAaI2vSfHG8

Schmitt, É.-E. Autobiographie virtuelle. Récupéré de https://www.eric-emmanuel-schmitt. com/musique.html

Steiner, G. (2003). Maîtres et disciples (P.-E. Dauzat trad.). Paris : Gallimard.

Viart, D., Vercier, B. (2008). La Littérature française au présent. Héritage, modernité, mutations. Paris : Éditions Bordas.

RÉSUMÉ : Dans plusieurs œuvres à éléments autobiographiques, Éric-Emmanuel Schmitt révèle la genèse musicale de sa vocation d'écrivain en soulignant le rôle de quelques compositeurs ou bien en décrivant la maïeutique pédagogique mise en œuvre par ses professeures de piano. L'objectif de cet article est d’étudier le rôle (trans)formateur de l'apprentissage musical et plus particulièrement de l'initiation à la musique de Mozart, Beethoven et Chopin. Guides spirituels et maîtres à penser, ces compositeurs ont marqué le parcours existentiel et artistique d'Éric-Emmanuel Schmitt et ont inspiré sa propre aventure esthétique. La rencontre salutaire avec Mozart a induit la naissance de l'amour spirituel et a engendré le processus de conversion scripturale et de transmission créatrice. Le disciple mozartien réinvente sa vocation et transpose dans son œuvre littéraire des particularités de la philosophie musicale de ses maîtres de sagesse en s'inscrivant dans une logique de filiation spirituelle et esthétique.

Mots-clés : Éric-Emmanuel Schmitt, disciple mozartien, guides spirituels, hommage aux grands compositeurs, rapports entre musique et littérature

\section{Eric-Emmanuel Schmitt and his masters of happiness: the convergence of literary vocation and musical inclination}

ABSTRACT: In some of his works, containing autobiographical elements, EricEmmanuel Schmitt reveals the musical origins of his vocation as a writer. He emphasizes the importance of several composers and describes the maieutic method used by his piano teachers. The paper studies the (trans)forming function of learning music, and more specifically the initiation to the works of Mozart, Beethoven and Chopin. Spiritual guides and maîtres à penser, these composers influenced Schmitt's 
existential and artistic trajectory and inspired his own esthetic adventure. The salutary encounter with Mozart caused the birth of spiritual love and enabled an acceptance of the writing vocation and initiation of the process of creative transmission. The Mozartian disciple reinvents his vocation and transposes in his literary works some features of the musical philosophy of his masters of wisdom and thus, perpetuates their esthetical and spiritual filiation.

Keywords: Eric-Emmanuel Schmitt, Mozartian disciple, spiritual guides, homage to the great composers, relations between music and literature 